\title{
MR Angiography Compared to Conventional Selective Angiography in Acute Stroke
}

\author{
Anna I. Tomanek, Shelagh B. Coutts, Andrew M. Demchuk, Mark E. Hudon, \\ William F. Morrish, Robert J. Sevick, Jessica E. Simon, Richard Frayne, \\ Alastair M. Buchan, Michael D. Hill
}

\begin{abstract}
Background and purpose: Accuracy of intracranial magnetic resonance angiography (MRA) and reliability of interpretation are not well established compared to conventional selective catheter angiography. The purpose of this study was to determine the accuracy of MRA in evaluation of intracranial vessels in acute stroke and transient ischemic attack (TIA) patients. Methods: Twenty-nine patients (seven females, 22 males; median age 53) with acute stroke or TIA were enrolled into the study. All patients underwent both MRA using a $3 \mathrm{~T}$ clinical magnet and conventional angiography within 48 hours. Median time between MRA and angiography was 263 minutes. Conventional angiography preceded MRA in 15 cases. Fourteen patients received thrombolysis during MRA or angiography. National Institutes of Health Stroke Scale scores were obtained prior to the MR exam. One neuroradiologist rated all conventional angiograms, which were used as gold standard. Five observers, blinded to conventional angiography results and all clinical information except symptom side, rated the MR angiograms. Kappa statistics were used to assess reliability; contingency tables were used to assess accuracy of non-enhanced and enhanced MRA. Results: Two hundred and fifty two intracranial vessels were assessed. Agreement between raters was good for both non-enhanced $(\kappa=0.50)$ and gadolinium-enhanced $(\kappa=0.46)$ images. There were a total of 26 vessels occluded by DSA. Overall, the non-enhanced MRA showed sensitivity of $84.2 \%$ ( $95 \%$ CI 60.4-96.6) and specificity of 84.6\% (95\% CI 78.6-89.4). The enhanced MRA showed sensitivity of 69.2 (95\% CI 38.6-90.9) and specificity of 73.6 (95\% CI 65.5-80.7). Conclusions: Magnetic resonance angiography is a good non-invasive screening tool for assessing intracranial vessel status in acute ischemic stroke. Angiography remains the gold standard for definitive assessment of the intracranial circulation.
\end{abstract}

RÉSUMÉ: Comparaison de l'angiographie par résonance magnétique à l'angiographie sélective conventionnelle pour l'évaluation de l'accident vasculaire cérébral aigu. Contexte et objectif: La précision de l'angiographie par résonance magnétique intracrânienne (ARM) et la fiabilité de son interprétation sont mal établies par rapport à l'angiographie sélective conventionnelle par cathéter. L'objectif de cette étude était de déterminer la précision de l'ARM pour l'évaluation des vaisseaux intracrâniens dans l'accident vasculaire cérébral (AVC) aigu et l'accident ischémique transitoire (AIT). Méthodes: Vingt-neuf patients, (sept femmes et 22 hommes - âge médian 53 ans) atteints d'AVC aigu ou d'AIT ont participé à l'étude. Tous les patients ont subi une ARM utilisant un aimant 3T et une angiographie conventionnelle en dedans de 48 heures de l'événement. Le temps médian entre l'ARM et l'angiographie était de 263 minutes. L'angiographie conventionnelle a précédé l'ARM chez 15 patients. Quatorze patients ont subi une thrombolyse pendant l'ARM ou l'angiographie. Le score au National Institutes of Health Stroke Scale a été déterminé avant de faire l'ARM. Un neuroradiologiste a lu tous les angiogrammes conventionnels qui ont été utilisés comme examen de référence. Cinq observateurs qui ignoraient les résultats de l'angiographie conventionnelle ainsi que l'information clinique sauf la latéralité des symptômes, ont lu les angiogrammes par résonance magnétique. La fiabilité a été évaluée au moyen de l'analyse statistique kappa; la précision de l'ARM non rehaussée et rehaussée a été évaluée au moyen de tables de contingence. Résultats: Deux cent cinquante-deux vaisseaux intracrâniens ont été évalués. La concordance entre les évaluateurs était bonne tant pour les images non rehaussées $(\mathrm{k}=0,50)$ que pour les images rehaussées au gadolinium $(\mathrm{k}=0,46)$. À l'angiographie numérique, 26 vaisseaux étaient occlus. En tout, l'ARM avait une sensibilité de 84,2\% (IC 95\%:60,4 à 96,6) et une spécificité de 84,6\% (IC 95\%: 78,6 à 89,4). L'ARM rehaussée avait une sensibilité de 69,2\% (IC 95\% : 38,6 à 90,9) et une spécificité de 73,6\% (IC 95\% : 65,5 à 80,7). Conclusions: L'angiographie par résonance magnétique est un bon outil de dépistage non effractif pour évaluer l'état des vaisseaux intracrâniens dans l'AVC aigu. L'angiographie demeure l'examen de référence pour l'évaluation de la circulation intracrânienne.

Can. J. Neurol. Sci. 2006; 33: 58-62

Magnetic resonance angiography (MRA) allows visualisation of both extra- and intra-cranial vessels in a fast and non-invasive manner and can play an important role in clinical decisionmaking. Assessment of the intra-cranial vessels ${ }^{1-5}$ has led to multiple improvements of the technique with a significant increase in the accuracy of intra-cranial MRA. Prior research has shown benefit of careful review of the 3D TOF source images
From the Seaman Family MR Research Centre (AIT, SBC, AMD, JES, RF, AMB), Foothills Medical Centre, Calgary Health Region, Department of Clinical Neurosciences (AIT, SBC, AMD, MEH, WFM, RJS, JES, RF, AMB, MDH) Department of Community Health Sciences (MDH), Department of Medicine (MDH), and Department of Radiology (MEH, WFM, RJS, RF),

University of Calgary; Calgary, $\mathrm{AB}$ Canada.

ReCEIVED JANUARY 4, 2005. ACCEPTED IN FINAL FORM SEPTEMBER 17, 2005. Reprint requests to: Michael D. Hill, Rm 1242A, Foothills Hospital, 1403 29th Street NW, Calgary, Alberta, T2N 2T9, Canada. 
along with maximum intensity projections (MIP) and collapsed MIPs. ${ }^{1,5}$ Source images are particularly helpful for the assessment of intra-cranial stenoses. While most previous studies have concentrated on the proximal intracranial vessels, a few studies have reported difficulty in evaluating peripheral vessel branches. ${ }^{1,2}$ Contrast-enhanced MRA ${ }^{4,5}$ improves conspicuity of vessels of small caliber and slow blood flow.

In the setting of acute ischemic stroke clinicians seek information about the status of intracranial vessels. Hyperacute neurovascular imaging is challenging for number of reasons: (i) information needs to be obtained quickly; (ii) patients suffering severe disabling stroke do not tolerate prolonged imaging time and; (iii) relatively short acquisition time limits achievable image quality.

The purpose of this study was to investigate the reliability and accuracy of intracranial MRA of both proximal and distal vessels compared to conventional angiography in the setting of acute ischemic stroke (AIS) and transient ischemic attack (TIA).

\section{Methods}

\section{Patients}

We reviewed our research imaging database to identify patients who had undergone MR angiography. All patients with AIS or TIA, who for clinical reasons, underwent both 3 T MRI and conventional angiography within 48 hours from one another were recruited. Written informed consent to the use of the imaging in research was provided by all patients or their surrogate before MR imaging and conventional angiography.

\section{MR Imaging Protocol}

Magnetic resonance studies were performed on a 3.0 Tesla MRI system (Signa; GE Medical Systems, Waukesha, WI) equipped with high speed gradients $(40-\mathrm{mT} / \mathrm{m}$ peak strength, $184-\mu$ s rise time) using a standard quadrature head coil. The acute stroke protocol included standard anatomic imaging, diffusion-weighted imaging (DWI), three dimensional time-offlight (3D TOF) MR angiography, and dynamic susceptibility contrast perfusion-weighted imaging (DSCPWI). ${ }^{5}$ Time-of-flight angiograms were collected before (non-enhanced) and after (enhanced) the perfusion imaging contrast injection $(20 \mathrm{ml}$ of MR contrast - Magnevist, Berlex, Wayne, $\mathrm{NJ}$ - at $5 \mathrm{ml} / \mathrm{s}$ ).

The imaging parameters for the conventional non-enhanced two-slab 3D TOF sequence were 24/3.3/15 (TR/TE/flip angle), acquisition bandwidth of $12.5 \mathrm{kHz}$, and a $240 \times 144 \times 46-\mathrm{mm}$ acquired volume with a $256 \times 192 \times 42$ acqusition matrix, which was then reconstructed to a 512 x 512 × 84 matrix. Total acquisition time was 2 minutes 48 seconds. Axial slabs were prescribed from skull base to the circle of Willis. Post-contrast 3D TOF MR angiography focused on visualizing the intracranial circulation and therefore the non-enhanced technique was modified. First, an inclined slab was used to image the intracranial vasculature including the distal ICA and the proximal vessels of the circle of Willis. Second, to maximize vessel signal intensity on post-contrast images, the TR and flip angle were increased. Post-contrast $\mathrm{MR}$ angiographic parameters were as follows: $32 / 3.4 / 35^{\circ}$ (TR/TE/flip angle) acquisition bandwidth of $12.5 \mathrm{kHz}$, and a $240 \times 144$ x 36-mm acquired volume with a $256 \times 192 \times 40$ acquisition matrix, which was then reconstructed to a 512 × 512 × 80 matrix. Total acquisition time was 4 minutes 9 seconds. Using the non-enhanced MRA source images at the circle of Willis, maximum intensity projection (MIP) images were generated at multiple projection angles every $12.9^{\circ}$ about the superior/inferior (S/I) axis. Axial collapsed MIP images of both non-enhanced and enhanced MRA volumes were also generated.

Twenty-four patients had both non-enhanced and enhanced MRA, four patients had only non-enhanced MRA, and one patient had only enhanced MRA. Four enhanced MRA studies were excluded because of incorrect slab position.

\section{Angiography protocol}

Conventional angiograms were performed in a standard manner starting with an arch aortogram followed by selective injections of the carotid and/or vertebral arteries. Biplane digital subtraction angiography was performed (LCA/LP Advantx Angiography unit, General Electric). Intracranial vessels were imaged with a minimum field of view of $15 \mathrm{~cm}$ in the anteroposterior plane and $11.4 \mathrm{~cm}$ in the lateral plane at a magnification factor of 2.6. A high resolution (1024 x 1024) matrix was used.

\section{$M R A$ review}

Film copies of axial collapsed MIP images and source images for both non-enhanced and enhanced 3D TOF, as well as nonenhanced MIP images were prepared for review. Five readers two neuroradiologists, one stroke neurologist, and two stroke fellows blind to conventional angiography results and all clinical information except symptom side scored all MRA films. Each observer was given both non-enhanced and enhanced films at the same time, although the non-enhanced were scored before the enhanced films. The following intra-cranial vessels and vessel segments: internal carotid artery (ICA); M1 middle cerebral artery (MCA); M2 MCA; M3 MCA; anterior cerebral artery (ACA); vertebral arteries; basilar artery and posterior cerebral artery (PCA) were scored as patent or occluded based on signal intensity. A vessel or vessel segment was considered occluded if there was absent flow on all of MRA source images, MIPs and collapsed MIPs, within each modality (with or without MR contrast).

\section{Angiography review}

One experienced neuroradiologist blinded to MRA findings reviewed all angiograms. He was given the information about symptom side, and also which procedure was performed first: MRA or conventional angiography. He was asked to score only that part of the conventional angiogram, which was closest in time to the MRA in order to minimize the likelihood of vascular changes that may have occurred in between. Identical to the MRA scoring method, the analogous vessel segments were rated as patent or occluded. This statement was considered a gold standard.

\section{Data analysis}

Kappa statistics were used to assess inter-observer reliability. Agreement was considered to be slight $(\kappa=0.0-0.2)$, fair $(\kappa=0.21$ $0.4)$, moderate $(\kappa=0.41-0.60)$, substantial $(\kappa=0.61-0.80)$, or 
almost perfect $(\kappa=0.81-1.00) .{ }^{6}$ Contingency tables were used to assess the accuracy of non-enhanced and enhanced 3D TOF MRA compared to conventional angiography. Magnetic resonance summary scores used for this comparison were the median of 5 raters which is equivalent to a 3 of 5 consensus. We did not include the posterior circulation data in this analysis because of a paucity of occlusions in this territory.

\section{RESULTS}

Twenty-nine patients ( 7 females, 22 males), aged 18 to 83 years (median 53 years) with acute stroke (26 cases) or TIA ( 3 cases) were enrolled. National Institutes of Health Stroke Scale (NIHSS) scores were obtained at patient presentation and prior to the MR exam by qualified examiners. Median NIHSS was 14 (range 0 to 25). Fourteen patients received thrombolysis during MRA or angiography. Angiography preceded MRA in 15 cases. Thirteen patients (45\%) had their two studies within 3 hours one from another. Median time between non-enhanced and enhanced MRA was $22 \mathrm{~min}$. Twenty-six occlusions were diagnosed during conventional angiography among 252 intra-cranial vessels assessed: 25 in the anterior ciruclation (ICA 8, M1-MCA 10, M2MCA 4, M3-MCA 3) and one in posterior circulation (PCA 1).

Overall agreement among 5 raters was moderate for both nonenhanced $\left(\mathrm{k}=0.50 \quad \mathrm{CI}_{95} \quad 0.47-0.53\right)$ and gadolinium-enhanced $\left(\kappa=0.46 \mathrm{CI}_{95} 0.37-0.58\right)$ images. A gradient of agreement existed such that agreement for the proximal vessels was excellent, while for the distal vessels, agreement was poorer (Table 1). The overall accuracy of non-enhanced MRA was $84.5 \%\left(\mathrm{CI}_{95} 0.79\right.$ $0.89)$ and for enhanced MRA 73.2\% $\left(\mathrm{CI}_{95} 0.65-0.80\right)$. The nonenhanced MRA showed sensitivity of $84.2 \%\left(\mathrm{CI}_{95}\right.$ 60.4-96.6) and specificity of $84.6 \%\left(\mathrm{CI}_{95} 78.6-89.4\right)$. The enhanced MRA showed sensitivity of $69.2\left(\mathrm{CI}_{95} 38.6-90.9\right)$ and specificity of $73.6\left(\mathrm{CI}_{95}\right.$ 65.5-80.7). This was not substantially changed by inclusion of dynamic cases where reperfusion occurred due to therapeutic intervention.

In five cases the vessel status was dynamic as re-perfusion was achieved between angiography and the MR imaging as the result of interventional therapy. Consequently, there was clear discrepancy in the scoring of the vessel status by MRA and conventional angiography. After excluding those cases the accuracy of MRA was very good including distal vessels (Table 2).

Although agreement among raters was not improved with contrast enhancement, we found a positive impact of contrast administration on vessel assessment. In the ICA, use of contrast

\section{Table 1: Inter-rater agreement for pre- and post contrast} MRA

\begin{tabular}{c|c|c}
\hline VESSEL & $\begin{array}{c}\text { PRE-CONTRAST } \\
\kappa(95 \% \text { LCI })\end{array}$ & $\begin{array}{c}\text { POST-CONTRAST } \\
\kappa(95 \% \text { LCI })\end{array}$ \\
\hline ICA & $0.92(0.81)$ & $0.7(0.58)$ \\
MCA-M1 & $0.53(0.44)$ & $0.55(0.45)$ \\
MCA-M2/M3 & $0.17(0.09)$ & $0.18(0.08)$ \\
\hline
\end{tabular}

Table 2: Sensitivity and specificity of pre-contrast MRA after removing "Dynamic" cases

\begin{tabular}{c|c|c|c}
\hline \multirow{2}{*}{ Vessel } & & Sensitivity & Specificity \\
\hline \multirow{2}{*}{ ICA } & Pre-gadolinium & $0.86(0.42-1)$ & $0.94(0.73-1)$ \\
& Post-gadolinium & $1(0.75-1)$ & $0.93(0.66-1)$ \\
M1 & Pre-gadolinium & $0.75(0.35-0.97)$ & $0.83(0.69-0.93)$ \\
& Post-gadolinium & $0.67(0.22-0.96)$ & $0.79(0.59-0.92)$ \\
M2/M3 & Pre-gadolinium & $0.80(0.28-0.99)$ & $0.69(0.53-0.82)$ \\
& Post-gadolinium & $0.50(0.07-0.93)$ & $0.87(0.69-0.96)$ \\
\hline
\end{tabular}

non-significantly raised sensitivity to $100 \%$, while in distal MCA (M2/M3 segments) a trend to improved specificity was observed $(\mathrm{p}=0.087$ ) (Figure 1). Similarly, level of training was relevant. The overall specificity of the neurologists' assessments of the post-contrast images was $0.77\left(\mathrm{CI}_{95} 0.58-0.90\right)$, while the analogous result of the neuroradiologists' was $1.00\left(\mathrm{CI}_{95} 0.88\right.$ 1.00). Overall, neurologists' accuracy ranged from $77-81 \%$ for pre- or post-contrast MRI with or without the dynamic cases. This compares to $86-91 \%$ for neuroradiologists. Accuracy was always equivalent or non-significantly higher for the contrastenhanced assessment.

\section{DISCUSSION}

Thrombolytic therapy remains the only proven therapeutic intervention with a potential to restore perfusion and reverse symptoms in patients suffering AIS. Imaging modalities (MR and CT) that are capable of rapidly identifying vessel occlusion causing symptoms of stroke, are indispensable in this scenario. While occlusion identification substantiates therapeutic efforts, any imaging protocol for AIS or TIA has specific restrictions and represents a trade-off between patient comfort, technical optimization and diagnostic accuracy.

The reliability of MRA has improved with technical innovation. Korogi et $\mathrm{al}^{2}$ noted in 1994 that each of the observers, participating in their study of unenhanced TOF MR angiography, interpreted several ICA occlusions or stenoses of greater than $50 \%$ as normal. Consequently the authors found MRA unreliable for the assessment of severe ICA stenoses. The addition of 3D TOF source images resulted in increased sensitivity to $100 \%$ for moderately and severely stenosed, as well as occluded vessels. ${ }^{7}$ Heiserman et $\mathrm{al}^{1}$ achieved good results $3 \mathrm{D}$ TOF source images were combined with projections and collapsed MIP images. Thus, the interpretation protocol must utilize all available images including MIP and source images to achieve the best diagnostic result.

Robinson et $\mathrm{al}^{8}$ described application of the multiple overlapping thin slab acquisition (MOTSA) in the evaluation of carotid artery. Multiple overlapping thin slab acquisition combines the advantages of two- and three-dimensional TOF 


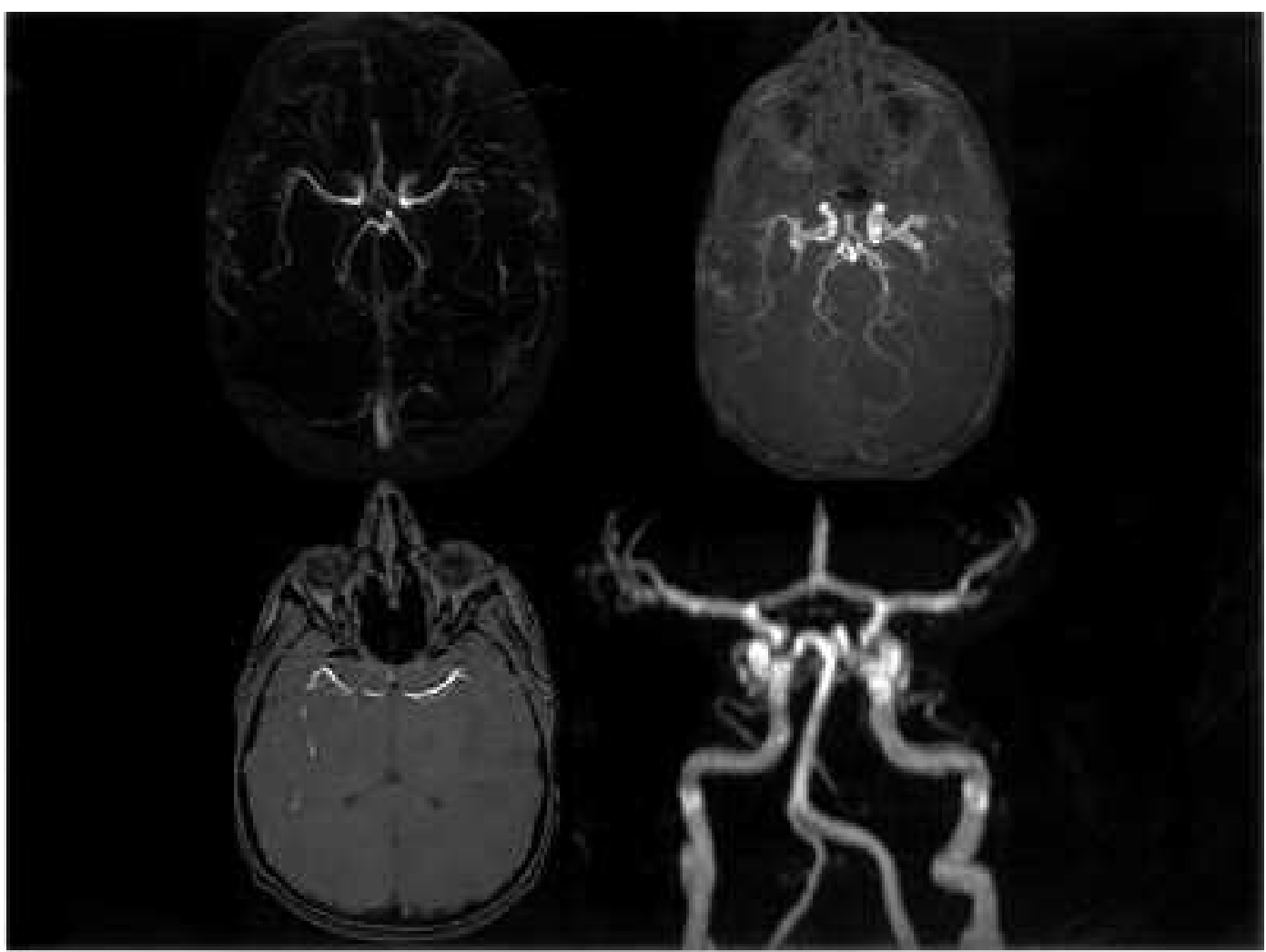

Figure: 3D TOF images of a 33-year-old male patient with left M2-MCA occlusion showing the MRA source image, maximum intensity projection (MIP), pre-contrast collapsed MIP, post-contrast collapsed MIP.

techniques: noise reduction and thin section detail with improved blood flow signal. However, early MR angiograms obtained using a MOTSA technique and postprocessed by the MIP algorithm displayed signal intensity variation, called venetian blind artifact. This artifact can be overcome by optimizing image acquisition parameters (flip angle, TR, and the slab excitation fraction), as well as post-processing algorithm, which results in a reduction of slab boundary artifact. A similar technique was used in this study.

Improved MRA techniques have been reported using higher matrix, overlapping slabs, a variable flip angle excitation (tilted optimized nonsaturating excitation - TONE), and magnetization transfer suppression (MTS), but this technique comes at the expense of a 60 - 90 min examinaiton time; such times are untenable in acute stroke. ${ }^{3}$ Even these significant technical improvements did not eliminate artifacts such as flow saturation, that result in relatively common false positive diagnoses of vessel stenoses, especially at bifurcations.

The advantages of a 3D TOF technique include signal-tonoise reduction and better delineation of small vessels. However, 3D-TOF is more sensitive to saturation resulting in poorer vessel definition. Contrast enhanced intracranial angiography has been explored and may be superior to non-enhanced studies. ${ }^{5,9}$ Loss of signal due to saturation of slowly flowing blood can be compensated by the $\mathrm{T} 1$ reduction achieved with paramagnetic extracellular contrast agents, such as gadopentetate dimeglumine. ${ }^{10}$ Other techniques suppressing soft tissue background such as magnetization transfer, optimization of imaging parameters, including TR, TE, and flip angle, have been explored and have been shown to improve imaging of the intracranial vessels.

Not all of the above techniques can be implemented at higher fields. In order to optimize our MRA protocol we have modified imaging parameters by using variable flip angle, increased TR and TE for post-contrast MRA (see Methods). For acquisition of non-enhanced 3D-TOF we have used 2 overlapping slabs, while for the enhanced MRA one inclined slab. Some techniques, like magnetization transfer are more difficult at higher fields in order to supress the soft tissue background.

We have determined the performance of MRA in evaluation of the intracranial vasculature. Exact grading of the possible vessel stenosis was not of paramount importance in acute stroke; therefore, we focused on recognising occlusion. Although image resolution remains better with conventional angiography, the raters were able to discern smaller vessel branches using MRA with satisfactory accuracy.

Similar to the other groups we have achieved moderate-tohigh sensitivity and specificity in the assessment of the ICA. Analogous results were achieved in evaluation of proximal MCA branches (M1 proximal and distal). Our group has previously explored the issue of contrast influence on evaluation of distal intracranial branches. ${ }^{5}$ A small proportion (3.3\%) of assessed vessels were less visible after contrast administration. This predominantly occurred in the P1 and P2 segments, which were 
not in scope of analysis of the present study, but was also observed in the anterior circulation. Explanation of this phenomenon remains unclear.

We have noted, that less experienced raters tended to overcall the occlusions, while experts (neuroradiologists) were more specific in their readings. Other authors ${ }^{11}$ have similarly raised the importance of training and experience in order to evaluate MRA accurately. Non-radiologists may not always be familiar with the technical limitations such as overestimation of the vessel stenosis using MIP algorithm. In our study it was important to pay attention to the boundaries of slab position: vessels not included in the slab appeared occluded on both source and postprocessed images.

There were limitations in our study. The major one was use of thrombolytic in $48 \%$ of cases, however, not using tPA for eligible patients would be unethical. In at least five cases, vessel status was clearly different between conventional angiography and MRA. Analysis has been conducted with and without these cases. It is possible that in a few cases vessel status changed even between pre- and post-contrast MRA, since the average time between the two was $20 \mathrm{~min}$. An additional drawback of the study was the lack or exclusion of contrast enhanced studies in eight cases. This obstructs full analysis of the impact of contrast administration on MRA.

The MRA is a reliable screening tool, when reviewed by expert readers familiar with MRI technique and possible artifacts, for the evaluation of intracranial vessels. However, angiography remains the gold standard for assessment of intracranial vessels and allows direct intra-arterial intervention. In the setting of acute stroke MRA is not evaluated in isolation. Other MRI sequences like DWI, FLAIR, and particularly PWI provide valuable information. Thus MRI provides comprehensive, non-invasive assessment of both the vessel and the status of ischemic tissue. Such information is invaluable in diagnosis and planning patient management.

\section{ACKNOWLEDGEMENTS}

The authors have no conflicts of interest to declare.

Supported in part by grants from the Heart and Stroke Foundation of Canada, the Alberta Heritage Foundation for Medical Research, CIHR, the Natural Sciences and Engineering Research Council of Canada, and the Canadian Stroke Network.

The 3.0 T MR Scanner used in this study was partially funded by the Canada Foundation for Innovation. The acute stroke imaging was paid for by the Alberta Foundation for Health Research.
Dr. Tomanek, Dr. Coutts and Dr. Simon were clinical fellows of the Alberta Heritage Foundation for Medical Research. Dr. Coutts was additionally supported by a Heart and Stroke Foundation of Canada (HSFC) fellowship. Dr. Hill was supported by the Heart \& Stroke Foundation of Alberta/NWT/NU and the Canadian Institutes for Health Research. Dr. Frayne is a HSFC Research Scholar and an AHFMR Medical Scholar.

\section{REFERENCES}

1. Heiserman JE, Drayer BP, Keller PJ, Fram EK. Intracranial vascular stenosis and occlusion: evaluation with three-dimentional timeof-flight MR angiography. Radiology. 1992;185:667-73.

2. Korogi Y, Takahashi M, Mabuchi N, et al. Intracranial vascular stenosis and occlusion: diagnostic accuracy of three-dimentional, fourier transform, time-of-flight MR angiography. Radiology. 1994; 193:187-93.

3. Stock KW, Radue EW, Jacob AL, Bao XS, Steinbrich W. Intracranial Arteries: prospective blinded comparative study of MR angiography and DSA in 50 patients. Radiology. 1995;195: 451-56.

4. Parker DL, Goodrich KC, Alexander AL, Buswell HR, Blatter DD, Tsuruda JS. Optimized visualization of vessels in contrast enhanced intracranial MR angiography. MRM. 1998;40:873-82.

5. Yang JJ, Hill MD, Morrish WF, Hudon ME, Barber PA, Demchuk AM, et al. Comparison of pre- and postcontrast 3D Time-of-flight MR angiography for the evaluation of distal intracranial branch occlusions in acute ischemic stroke. Am J Neuroradiol. 2002;23:557-67.

6. Landis JR, Koch GG. The measurement of observer agreement for categorical data. Biometrics. 1977;33:159-74.

7. Korogi Y, Takahashi M, Nakagawa T, Mabuchi N, Watabe, et al. Intracranial vascular stenosis and occlusion: MR angiographic findings. Am J Neuroradiol. 1997;18:135-43.

8. Robinson RO, Blatter DD, Parker DL, Barney WW, Perry DM, Goodrich KC. Reduction of slab boundary artifact with multiple overlapping thin slab acquisition in MR angiography of the cervical carotid artery. J Magn Reson Imaging. 1994;4:529-35.

9. Willig DS, Turski PA, Frayne R, Graves VB, Korosec FR, et al. Contrast-enhanced 3D DSA of the carotid artery bifurcation: preliminary study of comparison with unenhanced 2D and 3D time-of-flight MR angiography. Radiology. 1998;208(2):447-51.

10. Yano T, Kodama T, Suzuki Y, Watanabe K. Gadolinium-enhanced 3D time of flight MR angiography. Acta Radiol. 1997;38:47-54.

11. Wardlaw JM, Lewis SC, Collie DA, Sellar R. Interobserver variability of magnetic resonance angiography in the diagnosis of carotid stenosis - effect of observer experience. Neuroradiology. 2002;44(2):126-32. 